Department of Otolaryngology,

University of Oulu, PO Box 5000,

FIN-90014, Finland

${ }^{2}$ Department of Medical

Microbiology, Kajaanintie 50, FIN-

90029 Oulu University Hospital,

Finland

Correspondence to: O-P Alho

opalho@cc.oulu.fi

doi: 10.1136/bmj.39140.632604.55

\title{
Tonsillectomy versus watchful waiting in recurrent streptococcal pharyngitis in adults: randomised controlled trial
}

\author{
Olli-Pekka Alho, professor in otolaryngology, ${ }^{1}$ Petri Koivunen, consultant in otolaryngology, ${ }^{1}$ Tomi \\ Penna, specialist in otolaryngology, ${ }^{1}$ Heikki Teppo, specialist in otolaryngology, ${ }^{1}$ Markku Koskela, chief \\ physician, ${ }^{2}$ Jukka Luotonen, chief physician
}

\section{ABSTRACT}

Objective To determine the short term efficacy and safety of tonsillectomy for recurrent streptococcal pharyngitis in adults.

Design Randomised controlled trial.

Setting Academic referral centre in Finland.

Participants 70 adults with documented recurrent episodes of streptococcal group A pharyngitis.

Intervention Instant tonsillectomy $(n=36)$ or remaining on waiting list as control $(n=34)$.

Main outcome measures Percentage change in the risk of an episode of streptococcal pharyngitis at 90 days. Rates of all episodes of pharyngitis and days with symptoms and adverse effects.

Results The mean (SD) follow-up was 164 (63) days in the control group and 170 (12) days in the tonsillectomy group. At 90 days, streptococcal pharyngitis had recurred in $24 \%(8 / 34)$ in the control group and $3 \%(1 / 36)$ in the tonsillectomy group (difference $21 \%$; $95 \%$ confidence interval $6 \%$ to $36 \%$ ). The number needed to undergo tonsillectomy to prevent one recurrence was 5 (3 to 16). During the whole follow-up, the rates of other episodes of pharyngitis and days with throat pain and fever were significantly lower in the tonsillectomy group than in the control group. The most common morbidity related to tonsillectomy was postoperative throat pain (mean length 13 days, SD 4).

Conclusions Adults with a history of documented recurrent episodes of streptococcal pharyngitis were less likely to have further streptococcal or other throat infections or days with throat pain if they had their tonsils removed.

Trial registration Clinical Trials NCT00136877.

\section{INTRODUCTION}

Group A streptococcal pharyngitis is an acute infection, mainly of the oropharynx, caused by Streptococcus pyogenes. ${ }^{12}$ Recommended treatment is antibiotics to prevent rheumatic fever and suppurative complications and to ameliorate symptoms and decrease contagion. ${ }^{1-3}$ Some patients experience multiple episodes of acute pharyngitis with results of culture positive for group A streptococci. Possible explanations for these episodes are new infections with group A streptococci, a streptococcal carrier having non-streptococcal infections, and failures of treatment. ${ }^{3}$

Traditionally, tonsillectomy has been used to prevent recurrent streptococcal throat infections. Yet according to a recent Cochrane review, there is no empirical evidence to show that it is effective in adults. ${ }^{4}$ The exact role played by infection of the palatine tonsils (the tissue removed in tonsillectomy) in streptococcal pharyngitis is unknown, as other pharyngeal lymphoid and soft tissues are often also infected. ${ }^{45}$ We conducted a randomised controlled trial on adults with documented recurrent episodes of streptococcal pharyngitis to determine the effects of tonsillectomy.

\section{METHODS}

\section{Participants}

From October 2001 to May 2005, consecutive patients referred for tonsillectomy because of recurrent episodes of streptococcal pharyngitis were screened for enrolment at an ear, nose, and throat department of a university hospital. All patients provided written informed consent.

The clinical criterion for study entry was three or more episodes of pharyngitis in six months or four episodes in 12 months. The symptoms and signs during the episodes had to be typical of streptococcal pharyngitis. ${ }^{1}$ In addition, these episodes had to be severe enough for the patient to seek medical attention and at least one episode had to be group A streptococcal infection proved by culture or rapid antigen test. Exclusion criteria were age under 15 years, history of peritonsillar abscess, ongoing antibiotic treatment for other illness, recurrence probably caused by non-compliance with treatment, major heart or airway disorder or bleeding diatheses that would make same day surgery unfeasible,${ }^{6}$ and residence outside the city of Oulu or the neighbouring eight communities.

\section{Study design}

We allocated patients to tonsillectomy or the waiting list (control). To avoid disparity between group sizes, we used replacement randomisation. If the disparity 
exceeded the preset criterion of six, we generated another randomisation list using simple randomisation until we achieved a balanced group size. ${ }^{7} \mathrm{~A}$ research assistant not involved in the assignment or care of the trial patients generated the randomisation sequence with a computer random number generator. The assistant concealed the allocation sequence from the investigators who enrolled the participants by putting the assigned treatments in sequentially numbered, opaque, sealed envelopes. These were opened sequentially only after an eligible participant had been found and informed consent obtained, after which a study nurse attached a self fastening slip of paper containing the patient's name to the official study logbook. A trial physician recorded the baseline data and status.

\section{Outcomes}

We tested the hypothesis that tonsillectomy in adults with recurrent streptococcal pharyngitis would reduce the short term risk of developing an episode of streptococcal pharyngitis, reduce the rate of all episodes and days with symptoms, and not cause excessive adverse effects.

The primary end point was the proportion of patients with an acute episode of group A streptococcal pharyngitis during the 90 days' follow-up, as determined by signs and symptoms of acute pharyngitis with a positive result of throat culture. The secondary end points were the percentage change in the proportions of patients with all episodes of pharyngitis at 90 days, the times to episodes, and the difference in the mean rates of episodes and days with symptoms during the whole follow-up. Patients recorded episodes and days with symptoms in diaries. We considered an episode to be at least two consecutive days with a sore throat. We recorded data on adverse effects related to tonsillectomy from the diaries and the patients' charts.

\section{Intervention}

After baseline data collection and randomisation, the participants were operated on as soon as possible (tonsillectomy group) or placed on the waiting list for tonsillectomy (control group). In the tonsillectomy group, the median time between the randomisation and the tonsillectomy was 13 days (interquartile range 8-21) for practical reasons. The operation was performed under general anaesthesia as day surgery. Four experienced ear, nose, and throat surgeons performed total extracapsular tonsillectomy using blunt or diathermy dissection. In the control group, the waiting times ranged from three to six months, during which time the patients did not receive any prophylactic treatment for their tonsillitis.

\section{Surveillance protocol}

We obtained background data and examined the patients at assignment. Both groups were followed up for at least 90 days after randomisation, but otherwise the time of the follow-up visit depended on the length of the waiting list for tonsillectomy in the control group (range 90-210 days) and was 150-180 days in the tonsillectomy group. At randomisation patients in the control group were given the first available date for the operation, the length of follow-up therefore being unrelated to the severity of symptoms.

The primary outcome was objective and based on the presence of group $A \beta$ haemolytic streptococci in the throat culture during acute pharyngeal symptoms. Patients were given a prepaid microbiological postal package and the phone number of the study nurse with instructions to order a new package immediately after using one. The package included equipment for taking a specimen (Transpocult, Orion Diagnostica, Helsinki, Finland). All participants were advised to visit their own general practitioner whenever they had acute symptoms suggestive of pharyngitis. The general practitioner would then take a culture sample from the pharynx and send it to Oulu University Hospital for analysis. The patients were given written instructions for their general practitioner about the study and illustrated information on how to obtain the culture sample - namely, from the surface of both tonsils or tonsillar fossae in the patients who had undergone tonsillectomy and the posterior pharyngeal wall. The patients were told that it was important to seek medical advice for their symptoms during the trial in exactly the same way they had done before the trial and that it was possible to have streptococcal pharyngitis after tonsillectomy.

We plated the swab on sheep blood agar, selecting the growth of streptococci, and incubated them at $35^{\circ} \mathrm{C}$ for 18-24 hours before reading. The plates were examined again at 48 hours. We used latex agglutination tests (streptococcal grouping kit, Oxoid, Unipath, Hampshire) to differentiate group A streptococcus from the other $\beta$ haemolytic streptococci. To identify streptococcal carriers, we obtained throat cultures at assignment when the patients were asymptomatic. We were able to serotype streptococcal isolates in case a carrier had a culture positive acute episode. All microbiological analyses were done blinded.

The secondary outcomes were based on the patients' symptoms. Patients used diaries to record their acute symptoms (fever, throat pain, cough, and rhinitis), episodes of pharyngitis, and visits to a doctor. Individuals with acute pharyngitis and positive throat cultures received treatment as prescribed by their own general practitioner. Patients in the tonsillectomy group also documented the duration of postoperative throat pain. At the follow-up visit, we collected the diaries and checked them for completeness.

\section{Statistical analysis}

We estimated that we needed to enrol 70 patients for the study to have a statistical power of $80 \%$ to detect an absolute difference in streptococcal recurrence rates of $25 \%$, given a 90 day recurrence rate of $25 \%$ in the control group and $0 \%$ in the tonsillectomy group. ${ }^{8} \mathrm{We}$ considered a two sided $\mathrm{P}$ value of 0.05 to indicate significance. For the primary and secondary end 
points, all participants were analysed on an intention to treat basis.

All data analyses were done according to a pre-established plan. Descriptive data are given as means (SD) or as medians with interquartile ranges. We used the Mann-Whitney $U$ test to compare continuous variables. We constructed survival curves, as they related to the treatment group, according to the Kaplan-Meier method, starting from the date of the randomisation. ${ }^{9}$ The differences between the groups were tested with the log rank test. ${ }^{10}$ We calculated the absolute difference and the $95 \%$ confidence intervals in the proportions of recurrence between the groups and the respective number needed to treat at 90 days. ${ }^{11}$ In the tonsillectomy group, we excluded from the risk time the individual recovery times immediately after tonsillectomy during which the patient had continuous throat pain (mean 13 days, SD 4), as reported by patients in their diaries.

\section{RESULTS}

\section{Patients}

The first patient underwent randomisation in October 2001, and the last participant completed the study in December 2005. We screened 298 candidates, 226 of whom were excluded, and two declined to participate. Most of those excluded had too few episodes of pharyngitis or had undocumented or non-streptococcal episodes or chronic tonsillitis. Of the 70 remaining patients who we enrolled, 34 were randomly assigned to the control group and 36 to the tonsillectomy group. All patients were seen at follow-up visits and were followed up for at least the scheduled 90 days, the mean lengths of follow-up being 164 days (SD 63) in the

Patients referred for tonsillectomy because of recurrent or chronic pharyngotonsillitis screened for eligibility $(n=298)$

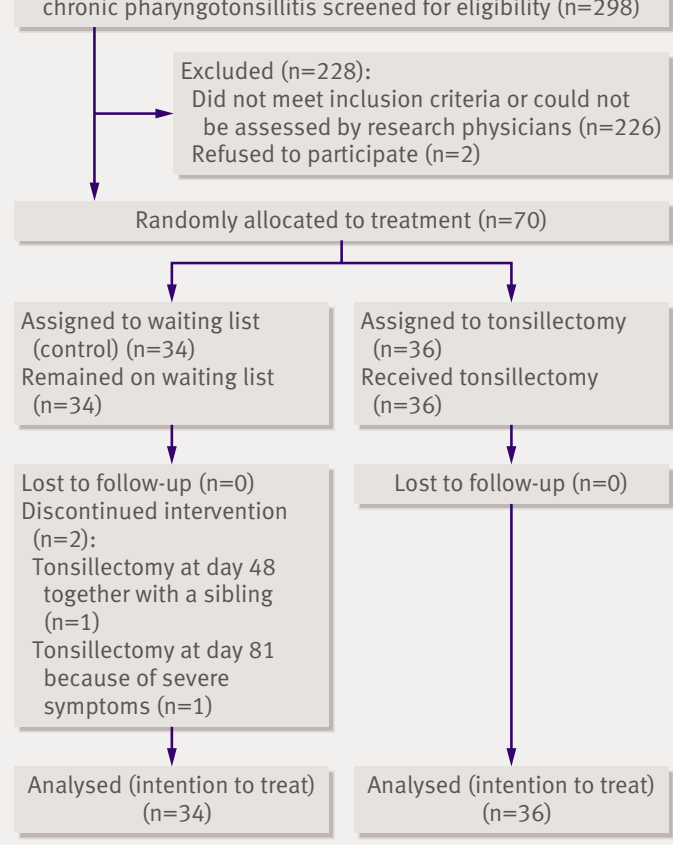

Fig 1 Enrolment and allocation of patients in study control group and 170 days (SD 12) in the tonsillectomy group. In the control group, one patient did not want tonsillectomy because of a lack of symptoms, but all the others were operated on after their follow-up. Tonsillectomy was done at the scheduled date in all except two cases, where the operation was done before the limit of 90 days (fig 1). One patient was operated on the same day as his sibling, who had a peritonsillar abscess, and the other had unbearable throat pain. There were no clinically important differences between groups in the baseline characteristics (table 1).

\section{Primary outcome}

At 90 days, according to the intention to treat in all randomised patients, eight patients (from 20 samples taken) in the control group and one (from five samples taken) in the tonsillectomy group had had an episode of group A streptococcal sore throat (difference 21\%, $95 \%$ confidence interval $6 \%$ to $36 \%$; number needed to treat 5, 3 to 16) (table 2). None of these patients were streptococcal carriers. This difference between the two groups was also evident in the time to the first episode (fig 2).

Table 1 | Demographic and baseline characteristics of adults with recurrent streptococcal pharyngitis according to allocation to waiting list (control) or immediate tonsillectomy. Figures are numbers (percentages) unless otherwise indicated

\begin{tabular}{lcc} 
Characteristic & $\begin{array}{c}\text { Control } \\
(\mathrm{n}=34)\end{array}$ & $\begin{array}{c}\text { Tonsillectomy } \\
(\mathrm{n}=36)\end{array}$ \\
Mean (SD) age (years) & $27(8)$ & $25(7)$ \\
\hline Female & $20(59)$ & $26(72)$
\end{tabular}

Tobacco use:

\begin{tabular}{lcc}
\hline By patient & $11(33)$ & $15(43)$ \\
\hline Someone else in the family & $9(27)$ & $14(40)$ \\
\hline History of allergy & $7(21)$ & $9(25)$ \\
\hline
\end{tabular}

Risk factors for pharyngitis:

\begin{tabular}{lcc}
\hline More than four people in family & $11(32)$ & $12(33)$ \\
\hline Similar infections in family & $5(15)$ & $4(11)$ \\
\hline Untreated caries & $4(12)$ & $6(17)$ \\
\hline Symptoms of gingivitis & $5(15)$ & $5(14)$ \\
\hline Use of same toothbrush >3 months & $11(32)$ & $11(31)$ \\
\hline
\end{tabular}

Mean (SD) No of previous episodes of acute pharyngitis*:

\begin{tabular}{lcc}
\hline During past 6 months & $3.3(1.5)$ & $3.5(1.3)$ \\
\hline During past 12 months & $4.8(2.1)$ & $5.1(1.9)$ \\
\hline Frequent throat pain & $20(59)$ & $23(64)$
\end{tabular}

Complications of pharyngitis:

\begin{tabular}{|c|c|c|}
\hline Joint symptoms & $3(9)$ & $3(8)$ \\
\hline Rheumatic fever & $0(0)$ & $0(0)$ \\
\hline \multicolumn{3}{|c|}{ Tonsils at baseline according to clinical assessment: } \\
\hline Merely large & $4(13)$ & $3(11)$ \\
\hline Chronically infected & $4(13)$ & $4(14)$ \\
\hline Scarred & $22(73)$ & $21(75)$ \\
\hline Carrier of group A streptococcus & $2(6)$ & $1(3)$ \\
\hline
\end{tabular}

*Exudative throat infection diagnosed by physician; according to inclusion criteria at least one episode had to be group A streptococcal infection proved by culture or rapid antigen test. 

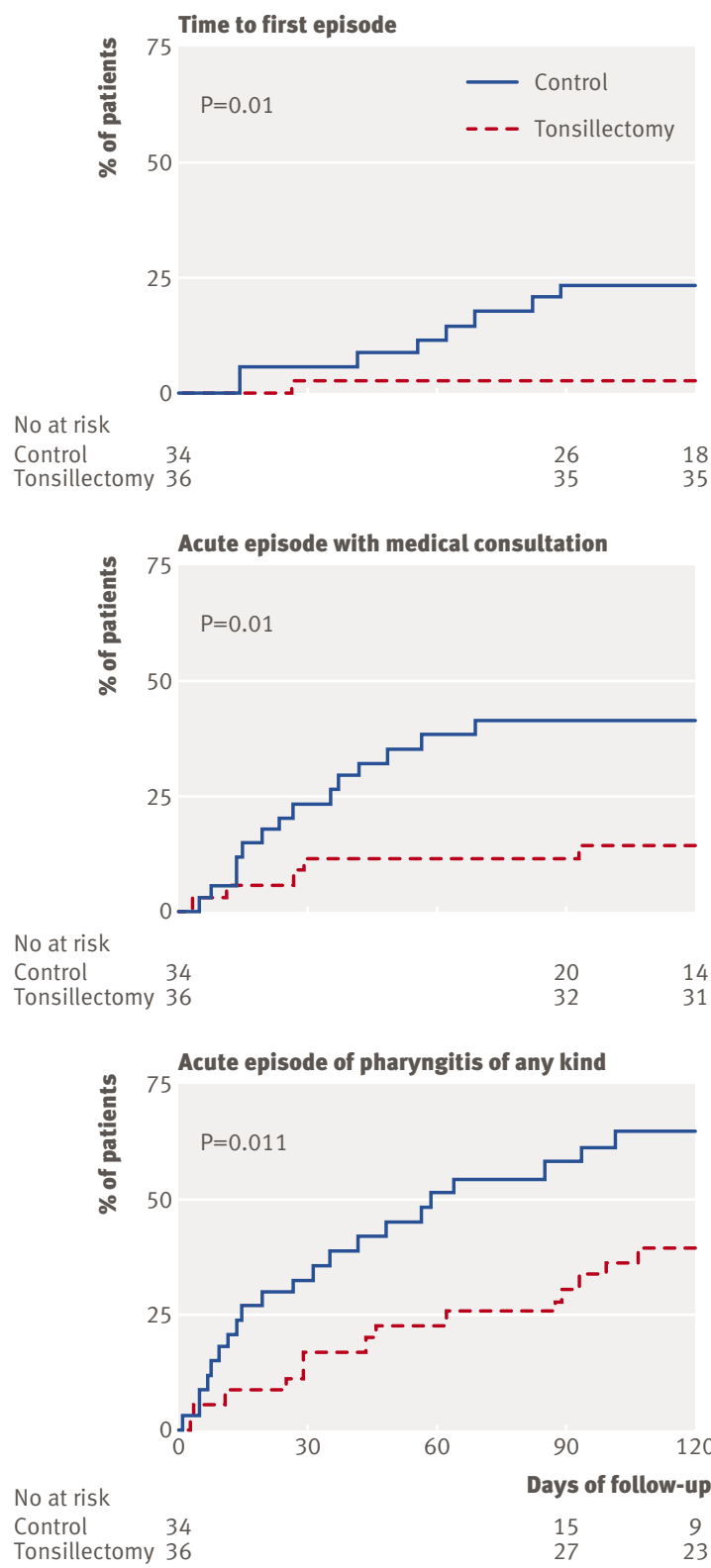

Fig 2 Times to first episode of group A streptococcal pharyngitis proved by culture, acute pharyngitis episode with medical consultation, and acute pharyngitis episode of any kind (differences between groups tested with log rank test)

Table 2 | Primary outcomes at three months in adults with recurrent streptococcal $A$ pharyngitis randomised to waiting list (control) or immediate tonsillectomy. Figures are numbers (percentages) of patients

\begin{tabular}{lccc} 
Outcome & $\begin{array}{c}\text { Control } \\
(\mathrm{n}=34)\end{array}$ & $\begin{array}{c}\text { Tonsillect- } \\
\text { omy }(\mathrm{n}=36)\end{array}$ & $\begin{array}{c}\% \text { difference } \\
(95 \% \mathrm{Cl})\end{array}$ \\
$\begin{array}{l}\text { Experienced episode of group A } \\
\text { streptococcal pharyngitis* }\end{array}$ & $8(24)$ & $1(3)$ & $21(6$ to 36) \\
\hline $\begin{array}{l}\text { Experienced episode of } \\
\text { pharyngitis with medical } \\
\text { consultation }\end{array}$ & $14(41)$ & $4(11)$ & 30 (11 to 49) \\
\hline $\begin{array}{l}\text { Experienced acute episode of } \\
\text { pharyngitis of any kind }\end{array}$ & $19(56)$ & $11(31)$ & $25(3$ to 48) \\
\hline
\end{tabular}

*Determined by signs and symptoms of acute pharyngitis with positive result of throat culture.

\section{Secondary outcomes}

At 90 days, compared with the tonsillectomy group, a significantly larger proportion of the control group had had acute episodes of pharyngitis, both with and without need for medical assessment (table 2). The times to these first episodes were significantly shorter in the control group than in the tonsillectomy group (fig 2). Similarly, the mean numbers of days with fever or throat pain, but not rhinitis or cough, during followup were significantly higher in the control group than in the tonsillectomy group (table 3).

\section{Adverse effects of tonsillectomy}

On average, the tonsillectomy operation required a one hour stay in the operating room, a one day stay in hospital, and a 13 days recovery period with postoperative throat pain (table 4). There were no serious adverse effects related to tonsillectomy. Two patients $(6 \%)$ had mild secondary bleeding 9 and 11 days after the operation.

\section{DISCUSSION}

\section{Principal findings}

Our trial supports the surgical removal of palatine tonsils to prevent immediate further episodes of group A streptococcal pharyngitis in adults with a documented history of recurrent episodes. We estimate that performing tonsillectomy on such adults would lead to one extra patient avoiding streptococcal infection during the following months for every five patients treated. Tonsillectomy also significantly reduced the short term risk of other episodes of pharyngitis with or without need for medical intervention and the mean number of days with fever or throat pain, but not with rhinitis or cough. The most important morbidity related to the operation was postoperative throat pain and a small risk of bleeding after the operation. Our results support the theory that palatine tonsils have an important role in recurrent streptococcal pharyngitis.

\section{Comparison with other studies}

A recent systematic review of randomised trials in children estimated that (adeno)tonsillectomy reduced the

Table 3 | Secondary outcomes at end of whole follow-up* in adults with recurrent streptococcal A pharyngitis randomised to waiting list (control) or immediate tonsillectomy. Figures are means (SDs)

\begin{tabular}{lccc} 
Outcome & $\begin{array}{c}\text { Control } \\
(\mathrm{n}=34)\end{array}$ & $\begin{array}{c}\text { Tonsillect- } \\
\text { omy }(\mathrm{n}=36)\end{array}$ & P value \\
$\begin{array}{l}\text { Medical consultations for } \\
\text { episodes of pharyngitis }\end{array}$ & $0.9(1.1)$ & $0.1(0.3)$ & 0.002 \\
\hline All episodes of pharyngitis & $2.1(2.3)$ & $0.6(0.9)$ & 0.001 \\
\hline Days with sore throat & $12.1(14.1)$ & $3.2(5.3)$ & 0.002 \\
\hline Days with fever & $2.8(3.9)$ & $0.6(1.5)$ & 0.01 \\
\hline Days with rhinitis & $7.6(11.9)$ & $6.3(7.1)$ & 0.55 \\
\hline Days with cough & $2.6(5.5)$ & $2.6(3.3)$ & 0.17 \\
\hline
\end{tabular}

*Mean length of follow-up 164 days (SD 63) in control group and

170 days (SD 12) in tonsillectomy group.

†Mann-Whitney U test.

$\ddagger$ Not including postoperative throat pain in tonsillectomy group. 
Table 4 Details and adverse effects of tonsillectomy and operative findings in adults with recurrent streptococcal $A$ pharyngitis randomised to waiting list (control) or immediate tonsillectomy. Figures are numbers (percentages) unless otherwise indicated

\begin{tabular}{|c|c|c|}
\hline Characteristic & $\begin{array}{l}\text { Control } \\
(n=34)\end{array}$ & $\begin{array}{l}\text { Tonsillect- } \\
\text { omy }(n=36)\end{array}$ \\
\hline \multicolumn{3}{|l|}{ Operation type: } \\
\hline Tonsillectomy & - & $34(94)$ \\
\hline Adenotonsillectomy & - & $2(6)$ \\
\hline \multicolumn{3}{|l|}{ Operative technique: } \\
\hline Blunt dissection & - & $12(33)$ \\
\hline Diathermy dissection & - & $24(67)$ \\
\hline \multicolumn{3}{|l|}{ Mean (SD) times* $(\min )$ : } \\
\hline In operating room & - & $51(15)$ \\
\hline Under general anaesthesia & - & $42(12)$ \\
\hline Operation & - & $16(8)$ \\
\hline Median (IQR) blood loss (ml) & - & $5(5-31)$ \\
\hline $\begin{array}{l}\text { Mean (SD) time with postoperative } \\
\text { throat pain (days) }\end{array}$ & - & $13(4)$ \\
\hline Postoperative haemorrhage & - & $2(6)$ \\
\hline $\begin{array}{l}\text { Retained in hospital for postoperative } \\
\text { pain }\end{array}$ & - & $1(3)$ \\
\hline \multicolumn{3}{|l|}{ Findings at operationł: } \\
\hline Scarred tonsils & $26(77)$ & $30(83)$ \\
\hline Extracapsular microabscesses & $5(15)$ & $5(14)$ \\
\hline Granulating infected tonsils & $1(3)$ & $1(3)$ \\
\hline Merely large tonsils & $1(3)$ & $0(0)$ \\
\hline \multicolumn{3}{|c|}{$\begin{array}{l}\text { IQR=interquartile range. } \\
\text { *Data missing in one patient. } \\
\text { †Mild (no transfusion needed) secondary bleeding treated with } \\
\text { electrocautery in outpatient clinic. } \\
\ddagger 33 \text { patients in control group underwent tonsillectomy at end of follow- } \\
\text { up. There were no significant differences between groups in operative } \\
\text { finding. }\end{array}$} \\
\hline
\end{tabular}

incidence of episodes of sore throat by 1.2 episodes a year and reduced school absence associated with sore throat by 2.8 days a year, differences regarded by the authors as clinically insignificant. ${ }^{12}$ The respective decreases we found after tonsillectomy in adults were significantly higher at 3.3 episodes and 20 days with sore throat. The reduction in days with sore throat would have been smaller if we had included the days with postoperative throat pain. The reasons for the smaller effect size found in children may be that parents of the most severely affected children refused to participate in the trial, a high number of children switched from the control group to the surgery group, and a large proportion were lost to follow-up, which may have resulted in an underestimation of the treatment effect. ${ }^{12}$ With more stringent eligibility criteria, however, the treatment effect might be greater in children. ${ }^{8}$ A recent Cochrane review found no similar trials in adults. ${ }^{4}$

\section{Strengths and weaknesses}

As we had waiting list controls we could not exceed our normal waiting time for tonsillectomy, which resulted in a relatively short follow-up period. However, we think that the immediate effect of tonsillectomy reflects its overall usefulness. Moreover, when we consider the objective outcomes in earlier trials, the effect of tonsillectomy has not depended markedly on the follow-up time. ${ }^{12}$ A substantial improvement over time in the rate of episodes of pharyngitis occurred in the control group during the follow-up, probably because of the natural course of the disease. This improvement, however, makes it unlikely that the patients in the control group would have reported negatively biased data in their diaries, indicating that the difference between the groups was because tonsillectomy was beneficial rather than any detrimental effect of remaining on the waiting list. The use of waiting list controls had advantages as well. We wanted to enrol severely affected patients, to keep them in their assigned groups, and to follow them up. With this method, the patients in the control group knew they were going to be operated on, and $97 \%(70 / 72)$ agreed to participate, only $6 \%(2 / 34)$ switched from the control group, and none were lost to follow-up.

We chose the objective outcome of prevention of group A streptococcal throat infection as our main end point to minimise information bias. In open trials, subjective outcomes may lead to an overestimation of the intervention effect because patients in the control group are more likely to report outcomes than patients in the intervention group. ${ }^{13}$ Although the variables affecting the accuracy of results of throat culture (swabbing technique, quality and duration of incubation, culture media) were similar in the groups and blinded, information bias may have occurred here as throat culture specimens were taken when the patient's sought medical advice. There are several reasons why we think that this does not jeopardise our results. We emphasised to the patients in both groups that they should seek medical assessment as usual. The proportion of positive culture results of the samples taken was still higher in the control group than in the tonsillectomy group (8/20 and $1 / 5$, respectively). Finally, the symptoms of streptococcal throat infection are similar before and after tonsillectomy. ${ }^{14}$ In view of the baseline characteristics and the fact that most eligible patients entered the trial, we consider that our results are generalisable to the population seen in otolaryngological outpatient clinics in Finland. ${ }^{15}$ Extracapsular complete tonsillectomy is a clear cut procedure, and the use of several surgeons and surgical techniques further increase the generalisability of our results.

\section{Implications}

According to our results, tonsillectomy is an effective alternative for adults with a documented history of recurrent episodes of pharyngitis. Naturally, the morbidity and complications related to the operation must be considered. The most common postoperative complications were sore throat and mild bleeding. Several other factors, such as risks of anaesthesia, otalgia, fever, dehydration, dental injuries, burns, and soft tissue injuries, have been described. ${ }^{16}$ These complaints are usually mild, but a small risk of even life threatening complications exists (recent reported mortality ranging from 1 in 16000 to 1 in 35000$).{ }^{16}$ Physicians and 


\section{WHAT IS ALREADY KNOWN ON THIS TOPIC}

Observational studies have suggested that tonsillectomy may be effective in preventing recurrent streptococcal throat infections in adults

Evidence from randomised controlled trials has been lacking

\section{WHAT THIS STUDY ADDS}

Adults who have documented recurrent episodes of streptococcal pharyngitis are less likely to have further streptococcal or other throat infections or days with throat pain during the months after tonsillectomy

The most important morbidity related to tonsillectomy was postoperative throat pain and a small risk of bleeding after the operation
3 Bisno AL, Gerber MA, Gwaltney JM Jr, Kaplan EL, Schwartz RH. Practice guidelines for the diagnosis and management of group $A$ streptococcal pharyngitis. Infectious Diseases Society of America. Clin Infect Dis 2002;35:113-25.

4 Burton MJ, Towler B, Glasziou P. Tonsillectomy versus non-surgical treatment for chronic/recurrent acute tonsillitis. Cochrane Database Syst Rev 2000;(2):CD001802.

5 Feinstein AR, Levitt $M$. The role of tonsils in predisposing to streptococcal infections and recurrences of rheumatic fever. N Engl J Med 1970;282:285-91.

6 Tewary AK. Day-case tonsillectomy: a review of the literature. J Laryngol Otol 1993;107:703-5.

7 Schulz KF, Grimes DA. Generation of allocation sequences in randomised trials: chance, not choice. Lancet 2002;359:515-9.

8 Paradise JL, Bluestone CD, Bachman RZ, Colborn DK, Bernard BS, Taylor FH, et al. Efficacy of tonsillectomy for recurrent throat infection in severely affected children. Results of parallel randomized and nonrandomized clinical trials. N Engl J Med 1984;310:674-83.

patients must decide whether these clinical benefits outweigh the risk of further morbidity and the risks involved in the operation.

We thank Tuomas Holma for treating some of the patients, Tuula Gehör and Pirkko Kokko (trial nurses), and Raili Puhakka (research assistant).

Contributors: 0-PA was primarily responsible for designing, initiating, and conducting the study and data analysis and drafting of the manuscript and is guarantor. TP and HT participated in conducting the study and critical review of the manuscript. PK, MK, and JL participated in the study design, conducting the study, data analysis, and critical review of the manuscript.

Funding: None

Competing interests: None declared.

Ethical approval: Oulu University hospital ethics committee.

1 Bisno AL. Acute pharyngitis. N Engl J Med 2001;344:205-11.

2 Cooper RJ, Hoffman JR, Bartlett JG, Besser RE, Gonzales R, Hickner JM, et al. Principles of appropriate antibiotic use for acute pharyngitis in adults: background. Ann Intern Med 2001;134:509-17.
9 Kaplan EL, Meier P. Nonparametric estimation from incomplete observations. J Am Stat Assoc 1958;53:457-81.

10 Mantel N. Evaluation of survival data and two new rank order statistics arising in its consideration. Cancer Chemother Rep 1966;50:163-70.

11 Altman DG, Andersen PK. Calculating the number needed to treat for trials where the outcome is time to an event. BM/ 1999;319:1492-5.

12 Van Staaij BK, van den Akker EH, van der Heijden GJ, Schilder AG Hoes AW. Adenotonsillectomy for upper respiratory infections: evidence based? Arch Dis Child 2005;90:19-25.

13 McLeod RS. Issues in surgical randomized controlled trials. World Surg 1999;23:1210-4.

14 Chamovitz R, Rammelkamp CH Jr, Wannamaker LW, Denny FW Jr. The effect of tonsillectomy on the incidence of streptococcal respiratory disease and its complications. Pediatrics 1960;26:355-67.

15 Mattila PS, Tahkokallio O, Tarkkanen J, Pitkaniemi J, Karvonen M, Tuomilehto J. Causes of tonsillar disease and frequency of tonsillectomy operations. Arch Otolaryngol Head Neck Surg 2001;127:37-44.

16 Randall DA, Hoffer ME. Complications of tonsillectomy and adenoidectomy. Otolaryngol Head Neck Surg 1998;118:61-8.

Accepted: 9 February 2007 